\title{
Progress of research into circular RNAs in urinary neoplasms
}

\author{
Bangbei Wan ${ }^{\text {Equal first author, 1 }}$, Bo Liu ${ }^{\text {Equal first author, } 2}$, Cai Lv ${ }^{\text {Corresp. } 1}$ \\ ${ }^{1}$ Department of Urology, Central South University Xiangya School of Medicine Affiliated Haikou Hospital, Haikou, China \\ 2 Laboratory of Developmental Cell Biology and Disease, School of Ophthalmology and Optometry and Eye Hospital, Wenzhou Medical University, \\ Wenzhou, China \\ Corresponding Author: Cai Lv \\ Email address: Ivcai815@163.com
}

Circular RNAs (circRNAs) are a large class of endogenous RNA that form a covalently closed continuous loop without 5' or 3' tails and are diffusely expressed in mammalian cells. Through the development of high-throughput sequencing, microarray, and bioinformatics analyses, recent studies have shown that the expression of circRNAs is dysregulated in human tumor tissues and cells, as well as in the blood of patients, and closely correlates with the development of tumors. circRNAs can regulate the progression of tumors through various mechanisms. An increasing number of studies have shown that circRNAs may play critical roles in the early diagnosis, targeted therapy, and prognostic prediction of cancer as biomarkers or therapeutic targets. This review briefly describes the definitions and functions of circRNAs, and the main content includes the most recent progress in research into their function, regulation, and clinical relevance to bladder, renal, and prostate cancers. We also provide some novel ideas regarding the treatment of these diseases. 


\section{Progress of research into circular RNAs in urinary neoplasms}

2

3 Bangbei Wan ${ }^{1 \&}$, Bo Liu ${ }^{2 \&}$, Cai Lv ${ }^{1}$

4

5 Affiliations: ${ }^{1}$ Department of Urology, Central South University Xiangya School of Medicine

6 Affiliated Haikou Hospital, Haikou, China; ${ }^{2}$ Laboratory of Developmental Cell Biology and

7 Disease, School of Ophthalmology and Optometry and Eye Hospital, Wenzhou Medical

8 University, Wenzhou, China.

9

$\&$ These authors contributed equally to this work and should be regarded as co-first authors.

12 Correspondence: Corresponding author: Cai Lv, Department of Urology, Central South 


\section{Abstract}

28 Circular RNAs (circRNAs) are a large class of endogenous RNA that form a covalently

29 closed continuous loop without $5^{\prime}$ or $3^{\prime}$ tails and are diffusely expressed in mammalian cells.

30 Through the development of high-throughput sequencing, microarray, and bioinformatics

31 analyses, recent studies have shown that the expression of circRNAs is dysregulated in human

32 tumor tissues and cells, as well as in the blood of patients, and closely correlates with the

33 development of tumors. circRNAs can regulate the progression of tumors through various

34 mechanisms. An increasing number of studies have shown that circRNAs may play critical roles

35 in the early diagnosis, targeted therapy, and prognostic prediction of cancer as biomarkers or

36 therapeutic targets. This review briefly describes the definitions and functions of circRNAs, and

37 the main content includes the most recent progress in research into their function, regulation, and

38 clinical relevance to bladder, renal, and prostate cancers. We also provide some novel ideas

39 regarding the treatment of these diseases.

41 Key words: circRNA; bladder cancer; renal cancer; prostate cancer 


\section{Introduction}

47 Noncoding RNA (ncRNA) includes the major classes of housekeeping and regulatory ncRNAs. Housekeeping ncRNAs include ribosomal RNA (rRNA), tRNA and small nuclear RNA (snRNA). Regulatory ncRNAs can be classified according to length $(<200$ or $>200$ bp), including small noncoding RNA that contain microRNA (miRNA), snRNA, piRNA, siRNA, and

51 long ncRNA (lncRNA). Circular RNAs (circRNAs; > 200 bp) are unusual endogenous

52 noncoding RNAs (Bolha et al., 2017) that were first reported by Nigro et al. (1991). They were

53 initially considered to be splicing errors with no function (Cocquerelle et al., 1993), but through

54 the recent development of high-throughput sequencing, microarray, and bioinformatics analyses,

55 recent studies have shown that circRNAs may play pivotal roles in the occurrence and 56 progression of disease, including pancreatic cancer (An et al., 2018), breast cancer (Zhao et al.,

57 2018), epilepsy (Gong et al., 2018), and cardiovascular diseases (Wang et al., 2017). The present 58 review mainly highlights the most recent progress in research related to the functions, regulation, 59 and clinical relevance of circRNAs in bladder cancer (BC), renal cell carcinoma (RCC), and 60 prostate cancer ( $\mathrm{PCa}$ ) (Table 1) and provides novel ideas for future investigations and the 61 treatment of these diseases.

\section{Survey methodology}


64 We systematically searched titles in the PubMed database using the term, "circular RNA"

65 combined with “cancer," "guidelines," “prostate cancer," “renal cancer", "bladder cancer,"

66 "database," "data," and "identification." Publications that were unrelated to human circular RNA

67 were excluded. We did not refine factors such as journal, publishing date, or journal impact

68 factors during our search.

\section{Definition of circRNAs}

70 circRNAs comprise a class of non-coding RNA that have different structures from linear RNAs,

71 such as lncRNA and miRNA, and form covalently closed, continuous stable loop structures

72 without 3' or 5' ends. Back-splicing or lariat-circulating events might play vital roles in the

73 formation of integral circRNA. Most circRNAs are exons that stem from intergenic regions, but

74 a few originate from intergenic, intronic, antisense or untranslated regions (Fu et al., 2018).

75 According to their biogenesis, circRNAs can be categorized as exonic (ecircRNAs), circular

76 intronic (ciRNAs), retained-intron or exon-intron (EIciRNAs), and intergenic (Bolha et al.,

77 2017). Most circRNAs are located in the cytoplasm; however, some also reside in subcellular

78 compartments such as exosomes and mitochondria (Tian et al., 2018; Szabo \& Salzman,

79 2016; Shao et al., 2017; Chen et al ., 2017; Zhang et al., 2019) (Fig. 1A). Intron length, exon

80 length, respective sequences, and RNA-binding proteins (RBPs) can affect the formation of

81 circRNAs (Fu et al., 2018). 


\section{Functions of circRNAs}

\section{4 circRNAs can function as miRNA sponges}

85 circRNAs harbor miRNA binding sites within their sequences and can directly bind to miRNA,

86 inhibit combinations between miRNA and 3'-untranslated regions (3'-UTR) of target genes, and

87 abolish the effects of miRNA on gene expression (Chen and Duan,2018; Li et al., 2018; Bian et

88 al., 2018). This is the primary mechanism involved in the circRNA regulation of gene expression.

89 circ-SFMBT2 can serve as a sponge for miR-182-5p to regulate CREB1 mRNA expression and

90 promote the proliferation of gastric cancer cells (Sun et al., 2018). circ-0000523 inhibits

91 colorectal cancer, and its overexpression attenuates the proliferation of cancer cells and induces

92 apoptosis. circ-0000523 can interact with miR-31 and inhibit the Wnt/ $\beta$-catenin signaling

93 pathway, and thus, it can regulate the growth of colorectal cancer cells (Jin et al., 2018). circ-

94 NEK6 functions as an oncogenic circRNA in thyroid cancer as it is significantly up-regulated

95 and can promote the proliferation and invasion of cancer cells. The results of mechanical trials

96 have suggested that circ-NEK6 increases FZD 8 mRNA expression, activates the wnt signaling

97 pathway by interacting with miR-370-3p, which subsequently promotes the progression of

98 thyroid cancer (Chen et al., 2018). In addition, Wang et al. (2018) found that circ-DOCK1 is

99 distinctly upregulated in oral squamous cell carcinoma (OSCC) cell lines and OSCC tissues, and

100 that circ-DOCK1 downregulation can induce apoptosis in OSCC cell lines. circ-DOCK1 can also

101 increase $B I R C 3$ expression by acting as a sponge for miR-196a-5p, and then participating in the

102 process of OSCC apoptosis. 


\section{Translation}

105

106

107

circRNA differs from linear RNA. Some circRNAs possess an open reading frame; hence, they might be able to code proteins or peptides. One study has suggested that a circRNA (220 nt) from the rice yellow mottle virus can encode a highly basic, 16-kDa protein (AbouHaidar et al., 2014). One 2017 study found that consensus $\mathrm{N}^{6}$-methyladenosine $\left(\mathrm{m}^{6} \mathrm{~A}\right)$ motifs and a single $\mathrm{m}^{6} \mathrm{~A}$ site are sufficient to drive translation initiation, and proved that protein can be translated by circRNAs in human cells. Further studies have indicated that the $\mathrm{m}^{6} \mathrm{~A}$-driven translation of circRNAs requires the initiation factor eIF4G2 and m6A reader YTHDF3. Methyltransferase METTL3/14 can enhance, whereas demethylase FTO can inhibit $\mathrm{m}^{6} \mathrm{~A}$-driven translation of circRNAs (Yang et al., 2017). Moreover, others have found that circRNAs with internal ribosome entry site elements (IRES) or prokaryotic ribosome-binding sites can encode peptides (Perriman and Ares, 1998; Chen and Sarnow, 1995).

\section{circRNAs as gene transcribers and expression regulators}

Some circRNAs that circulate with introns "retained" between exons are called intron-containing circRNAs, such as ciRNA and EIciRNA, which mainly reside in the nucleus, interact with U1 snRNP, and enhance the transcription of their parental genes in a cis-acting manner ( $\mathrm{L} i$ et al., 2015). In addition, some circRNAs, such as circ-ankrd52 and circ-sirt7, can accumulate at the 
122

123

124 125 126

127

transcriptional sites of host genes, interact with RNA polymerase II (pol II) complexes, and then regulate the transcription of the parental genes (Chen, 2016). In addition, the synthesis of backspliced circRNAs competes with pre-mRNA splicing and leads to lower levels of linear mRNA that result in regulated gene expression (Ashwal-Fluss et al., 2014).

\section{Protein binding}

circRNAs can bind, store, sequester, and interact with proteins to regulate the expression and translation of genes. Many circRNAs interact with RNA binding proteins (RBPs). For example, the circRNA, circ-Mbl, and its flanking introns have conserved muscleblind (MBL) protein binding sites that firmly and specifically bind MBL. Modulating MBL levels significantly affects the biosynthesis of circ-Mbl (Ashwal-Fluss et al., 2014). Du et al. (2016) reported that high levels of circ-Foxo3 expression in non-cancer cells are associated with cell cycle progression. More concretely, aberrant circ-Foxo3 expression represses cell cycle progression by binding to the cell cycle proteins cyclin-dependent kinase $2(C D K 2)$ and cyclin-dependent kinase inhibitor 1 $(P 21)$, which results in the formation of a ternary complex and subsequently blocks cell cycle progression. Chen et al. (2019) found high levels of circ-AGO2 and human antigen R (HuR) expression and associated them with poor outcomes among patients with gastric cancer. circAGO2 promotes the growth, invasion, and metastasis of cancer cells in vitro and in vivo. The mechanisms involve physical interactions between circ-AGO2 and HuR protein to promote its activation and enrichment on the 3 '-untranslated region of target genes. This in turn results in 
142 decreased circ-AGO2 binding and the inhibition of gene silencing modulated by circ-

143 AGO2/miRNA that correlates with cancer progression.

\section{circRNAs and bladder cancer (BC)}

$146 \mathrm{BC}$ is the most prevalent among malignant tumors of the urinary system. The incidence of $\mathrm{BC}$ is

147 the highest among urogenital tumors in China, and it is second only to PCa in Western countries.

148 The incidence of $\mathrm{BC}$ in males is 3 to 4 -fold higher than that in females (Bray et al., 2018), and it

149 increases annually, with the incidence reaching a peak at ages between 50 and 70 years. Hence,

150 biomarkers are urgently needed to diagnose and treat BC. circ-RNA shows considerable

151 potential to serve as such biomarkers. Many studies have indicated that circRNAs are involved in

152 the occurrence and progression of BC through multiple mechanisms.

\section{Inhibitor activities}

circRNAs function as sponges for miRNA to upregulate target gene expression and inhibit the progression of BC (Fig. 1B). circ-HIPK3 is a circ-RNA derived from Exon 2 of the HIPK3 gene (Zheng et al., 2016). Silencing circ-HIPK3 can significantly inhibit the growth of human cells by directly binding to and inhibiting miR-124 activity (Zheng et al., 2016). Dysregulated circHIPK3 expression correlates with the occurrence and progression of human diseases (Cao et al., 2018; Yu et al., 2018) and circ-HIPK3 functions differ among diseases. Li et al. (2017) found 
161

162

163

164

165

166

167

168

169

170

171

172

173

174

175

176

177 that circ-HIPK3 is significantly decreased in BC tissues and cell lines, while it negatively correlates with $\mathrm{BC}$ grade, invasion, and lymph node metastasis. The overexpression of circHIPK3 effectively inhibits the migration, invasion, and angiogenesis of $\mathrm{BC}$ cells in vitro and suppresses BC growth and metastasis in vivo. Mechanistic studies have shown that circ-HIPK3 harbors two vital binding sites for miR-558 and can abundantly sponge miR-558 to suppress the expression of HPSE, VEGF, MMP9, thereby suppressing the progression of $\mathrm{BC}$. Therefore, circHIPK3 might be a novel biomarker for treating BC. Chi et al. (2019) reported that circ-000285 derived from HIPK3 inhibits BC. Analysis using quantitative real-time polymerase chain reaction (qRT-PCR) has shown that circ-000285 is downregulated in BC tissues, cell lines, and sera derived from patients with BC. In addition, the expression of circ-000285 is decreased almost three-fold among patients who are resistant to cisplatin compared with those who are cisplatin-sensitive. Additionally, circ-0000285 is associated with tumor size, differentiation, lymph node metastasis, distant metastasis, and TNM stage. Collectively, these findings indicate that circ-000285 can serve as a biomarker for BC diagnosis and chemotherapy, as well as a prognostic predictor. circ-BCRC4 is an important inhibitor of $\mathrm{BC}$ because its overexpression attenuates cancer cell proliferation and induces apoptosis. circ-BCRC4 also interacts with miR101 to downregulate EZH2 expression and induce cancer cell apoptosis (Li et al., 2017).

circRNAs also function as sponges of miRNAs to upregulate target gene expression and inhibit BC progression (Fig. 1B). For example, recent studies suggest that circ-ITCH expression is significantly downregulated in several cancer cell lines and tumor types (Luo et al., 2018; Wang 
181 182 183 184 185

et al., 2018). Enforced circ-ITCH expression can significantly suppress BC cell proliferation, migration, invasion, and metastasis, as well as induce apoptosis and G1/S cell cycle arrest in vitro and in vivo. Mechanistically, circ-ITCH enhances the expression of miR-17, miR-224 target gene $p 21$ and PTEN by "sponging” miR-17 and miR-224, which suppresses the aggressive biological behavior of BC (Yang et al., 2018). Xie et al. (2018) found that low levels of circBCRC-3 are expressed in BC tissues and cell lines, and that enhanced circ-BCRC-3 expression can attenuate $\mathrm{BC}$ cell proliferation in vitro and in vivo. Mechanistically, circ-BCRC-3 enhances the expression of miR-182-5p target gene cyclin-dependent kinase inhibitor 1B ( $p 27)$ by "sponging" miR-182-5p, which attenuates the aggressive biological behaviors of BC. Methyl jasmonate $(\mathrm{MJ})$, which has anticancer effects, can suppress $\mathrm{BC}$ progression by increasing circBCRC-3 expression. Hence, circ-BCRC-3 functions as a tumor inhibitor that suppresses BC progression through the miR-182-5p/p27 axis, which would be a novel target for BC therapy. circ-UBXN7 is also a novel supressor of BC, and miR-1247-3p is a carcinogenic factor, of which B4GALT3 is a target. Recent findings suggest that circ-UBXN7 is significantly downregulated in $\mathrm{BC}$ tissues compared with matched non-tumor tissues, and that circ-UBXN7 expression is related to the pathological stage, grade, and prognosis of BC. Others have shown that circUBXN7 directly binds miR-1247-3p, and can reverse the oncogenic effects induced by miR1247-3p. Mechanistically, circ-UBXN7 can function as a competitive endogenous RNA (ceRNA) of miR-1247-3p that enhances $B 4 G A L T 3$ expression, consequently inhibiting BC cell viability and invasion. Consequently, the circ-UBXN7-miR-1247-3p-B4GALT3 regulatory network should be a potential target for treating BC (Liu et al., 2018). Liu et al. (2018) reported that circ- 
202 FNDC3B also functions as a tumor suppressor in BC. circ-FNDC3B is a highly stable and

203 cytoplasmic circRNA derived from exons 5 and 6 of the $F N D C 3 B$ gene locus that is expressed at

204 low levels in BC cell lines and tissues, and associated with pathological T stage, grade,

205 lymphatic invasion, and the overall survival rates of patients. Functional experiments have

206 shown that overexpressed circ-FNDC3B significantly inhibits the proliferation, migration,

207 invasion, growth and lymphatic metastasis of tumors both in vitro and in vivo. Mechanical

208 experiments have shown that miR-1178-3p is a carcinogenic factor that targets the 5'UTR of

$209 G 3 B P 2$. circ-FNDC3B can function as a miR-1178-3p sponge that directly binds to miR-1178-3p

210 to regulate the expression of G3BP2, inhibit the SRC/FAK signaling pathway, and suppress the

211 development of BC.

\section{Promoter activities}

circRNAs are not only inhibitors but also promoters of BC. circRNA can elevate target gene expression by combining with miRNA and thus promote BC progression (Fig. 1B). Zhong et al. (2016) used microarrays to determine circRNA expression profiles in $\mathrm{BC}$ tissues and found that circ-TCF25 is upregulated. Furthermore, circ-TCF25 overexpression can downregulate miR103a-3p and miR-107, increase CDK6 expression and promote $\mathrm{BC}$ cell proliferation and migration in vitro and in vivo. Hence, circ-TCF25 might be a novel promising marker for BC. circ-BPTF is a novel circRNA derived from $B P T F$ exons, and it is significantly upregulated in BC, compared with adjacent normal tissues and cell lines. Patients who express more circ-BPTF 
222 have higher BC tumor grades and poorer prognoses. Functionally, circ-BPTF knockdown

223 reduces tumor progression in vitro and in vivo. Mechanistically, mimics of miR-31-5p, which is

224 a target miRNA of circ-BPTF, can partially reverse the effects of circ-BPTF. Furthermore,

$225 R A B 27 A$ is a target of miR-31-5p, and circ-BPTF weakens the anti-oncogenic effects of miR-31-

$2265 \mathrm{p}$ and consequently promotes $R A B 27 A$ expression. Therefore, attenuating circ-BPTF expression

227 might be a promising direction to pursue as a strategy for treating BC (Bi et al., 2018). Zeng et al.

228 (2019) found that levels of circ-VANGL1 BC are higher in BC than in adjacent normal tissues.

229 Functionally, silencing circ-VANGL1 significantly attenuates BC cell proliferation, cell cycle,

230 migration, and invasion in vitro and suppresses tumor growth in vivo. Mechanistically, circ-

231 VANGL1 directly binds to miR-605-3p. The interaction between circ-VANGL1 and miR-605-3p

232 inhibits miR-605-3p expression, by elevating that of VANGL1 that facilitates BC progression.

233 Sun et al. (2019) reported that circ-0058063 is upregulated in BC tissues, and that silencing circ-

2340058063 attenuates the migration capacity of cancer cells, increases cell apoptosis rates, and

235 arrests cells at the G0/G1 phase in vitro, thereby suppressing tumor growth in vivo.

236 Mechanistically, circ-0058063 can negatively modulate miR-145-5p as a sponge, and elevate

$237 C D K 6$ expression to promote $\mathrm{BC}$ progression.

238 Microarray analysis has shown that the circRNA, circ-MYLK, is significantly upregulated in BC.

239 Levels of circ-MYLK are associated with the progression of BC stage and grade. Functionally,

240 circ-MYLK overexpression noticeably enhances BC cell invasive and migratory capabilities.

241 Moreover, upregulating circ-MYLK can promote the epithelial-mesenchymal transition (EMT) 
242 of BC. Mechanistically, circ-MYLK overexpression enhances the expression of CD31, S100A4,

243 VEGFA, snail, N-cadherin, vimentin, Ras, p-RAF-1, p-MEK1/2, pERK1/2, and suppresses that

244 of E-cadherin and ZO-1. circ-MYLK functions as a ceRNA for miR-29a, thus activating

245 VEGFA/VEGFR2 and the downstream Ras/ERK signaling pathway to exert these effects (Zhong

246 et al., 2017). Huang et al. (2018) identified circ-0000144 as a novel oncogene in BC, because its

247 expression is significantly upregulated in BC, compared with adjacent non-tumor tissues, and

248 high levels of circ-0000144 expression are associated with a poor prognosis. Functionally, circ-

2490000144 knockdown attenuates BC cell proliferation and invasion in vitro, and reduces tumor

250 volumes in vivo. Mechanistically, circ-0000144 is a sponge for miR-217, and RUNX2 is a target

251 of miR-217. circ-0000144 enhances $R U N X 2$ expression by repressing miR-217 and thus exerts

252 oncogenic activities in BC.

253 circ-UVRAG is derived and cyclized by some exons from the $U V R A G$ gene. A recent study

254 indicated that circ-UVRAG is up-regulated in BC lines. Functionally, silencing circ-UVRAG

255 suppresses tumor formation and metastasis. Mechanistically, miR-223 is a downstream binding

256 target of circ-UVRAG, and miR-223 can target FGFR2. Downregulated circ-UVRAG promotes

257 miR-223 expression, which weakens FGFR2 expression in BC. Therefore, circ-UVRAG might

258 serve as a good diagnostic biomarker of BC, and contribute to targeted BC therapy (Yang et al.,

259 2019). Overall, the above evidence suggests that circRNAs have the potential to serve as novel

260 biomarkers for the diagnosis, therapy, and prognostic prediction of BC.

261 At present, these studies have mainly investigated conventional functions (ceRNA mechanisms) 
262 of circRNAs in BC. Unconventional functions of circRNAs, such as translation and protein

263 binding, should be further explored. Defining these functions will contribute to a deeper

264 understanding of the mechanisms underlying the involvement of circRNAs in cancer

265 development.

\section{circRNA and renal cancer}

Renal cancer also is a common urological tumor that was responsible for over 403,000 new diagnoses in 2018 and an estimated 175,000 deaths worldwide (Bray et al., 2018). Therefore, mortality. circRNAs play major roles in RCC (Xiong et al., 2019) (Fig. 1B). showed that circ-0001451 is significantly down-regulated in ccRCC tissues, and that it correlates with the clinicopathological features (including clinical stage, tumor stage, lymph node, and metastasis) and overall survival of patients with ccRCC. Functional experiments have confirmed that circ-0001451 knockdown promotes the proliferation of ccRCC cell lines and inhibits apoptosis in vitro, indicating its potential as novel diagnostic biomarker of ccRCC and a potential target for ccRCC treatment. However, the mechanism of ccRCC suppression requires further investigation. Estrogen receptor beta $(\mathrm{ER} \beta)$ is a carcinogenic factor in ccRCC. Increased 
281 ER $\beta$ expression is associated with a more advanced stage and a poor prognosis for patients with

282

283

284

285

286

287

288

289

290

291

292

293

294

295

296

297

298

299

300

ccRCC. Mechanistically, ER $\beta$ represses circ-ATP2B1 expression by inhibiting its host gene

ATP2B1 and directly binding to the ERE (-1765 to $-1760 \mathrm{nt})$ on the 5'-promoter of ATP2B1.

Moreover, ER $\beta$-circ-ATP2B1 might function by interacting with miR-204-3p, resulting in decreased miR-204-3p and increased FN1 mRNA expression that promotes ccRCC progression (Han et al., 2018). Wang et al. (2017) reported that androgen receptors (AR) interact with circHIAT1 and promote ccRCC development. Overall survival of is worse among patients with ccRCC who express low, compared with high levels of circ-HIAT1. Functionally, $A R$ promote ccRCC cell proliferation, migration and invasion. Mechanistically, $A R$ suppress circ-HIAT1 expression by inhibiting its host gene (HIAT1) at the transcriptional level and directly binding to the ARE on the HIAT1 promoter, regulating miR-195-5p/29a-3p/29c-3p expression, and thereby enhancing CDC42 mRNA expression to promote ccRCC progression. In summary, the above evidence suggests that circRNAs could serve as prognostic biomarkers and therapeutic targets for ccRCC. Many circRNAs exist in RCC, but only a few have been investigated. The manifold functions and mechanisms of circRNAs in RCC should be further explored in depth. In addition, conventional functions such as ceRNA mechanisms and unconventional functions such as translation and protein binding warrant further investigation.

\section{circRNA and prostate cancer (PCa)}

$\mathrm{PCa}$ is frequently diagnosed in elderly men. One report has indicated that about 1.3 million new 
301

302

303

304

305

306

307

308

309

310

311

312

313

314

315

316

317

318

319

320

321

diagnoses of PCa and 359,000 associated deaths will occur worldwide during 2018 (Bray et al., 2018), and that morbidity and mortality rates will continue to increase. circRNAs play vital roles in PCa development (Fig. 1B). The relative mRNA expression of circRNAs can be modulated by upstream or downstream factors to regulate $\mathrm{PCa}$ progression. Notably, miR-145 is a tumor suppressor in cancer, He et al. (2018) found using microarray analysis that miR-145 overexpression promotes the expression of circRNA-101981, circRNA-008068, and circRNA406557, and suppresses that of circRNA-101996 and circRNA-091420 in PCa LNCaP cells. Therefore, circRNAs can be regarded as regulatory factors in PCa development. circMYLK is not only a cancerogenic factor in BC but also in PCa. Dai et al. (2018) found significantly upregulated circ-MYLK expression in PCa tissues and cancer cell lines. Functionally, upregulated circRNA-MYLK significantly promotes tumor cell proliferation, invasion, and migration while decreasing cell apoptosis, whereas circ-MYLK knockdown reverses these processes. Mechanistically, circ-MYLK promotes PCa cell proliferation, colony formation, invasion, and migration by downregulating miR-29a expression. Kong et al. (2017) found high expression levels of circ-SMARCA5 in PCa samples compared with matched noncancerous prostate tissues, indicating that circ-SMARCA5 could be an oncogenic circRNA. Functional experiments have shown that silencing circ-SMARCA5 in PCa cells suppresses proliferation, increases the amount of cells in G1 phase, decreases that in S phase, and elevates apoptosis rates. However, the mechanisms of circ-SMARCA5 in PCa have not been investigated in detail. Presently, studies into the roles of circRNA in PCa are scarce, and further detailed investigations into the mechanisms of circRNAs are required because circRNAs have the potential to serve as 
322 therapeutic and prognostic targets of PCa.

323

324 Conclusion

325 circRNA expression markedly differs between cancer and healthy cells. circRNA function as

326 oncogenic factors or tumor suppressors that participate in cancer occurrence and progression

327 through a multitude of mechanisms. Dysregulated circRNA expression is inextricably associated

328 with cancer development. The expression of circRNAs correlates with clinical stage, tumor stage,

329 lymph node metastasis, and the prognosis of patients with cancer. circRNAs functionally

330 influence the proliferation, invasion, migration, cell cycle progression, apoptosis, and drug

331 resistance of cancer cells. circRNAs mechanistically act as sponges of miRNAs to indirectly

332 regulate target gene expression. Moreover, circRNAs can function upstream as proteins that

333 regulate target gene expression. circRNAs participate in cancer development through all these

334 mechanisms. Therefore, measures are needed to decrease cancerogenic circRNAs and/or enhance

335 the expression of circRNA tumor-suppressors as novel targets of cancer therapy.

References

Bolha L, Ravnik-Glavač M1, Glavač. 2017. Circular rnas: biogenesis, function, and a role as possible cancer biomarkers. International Journal of Genomics 2017: 6218353. DOI $10.1155 / 2017 / 6218353$.

Nigro JM, Cho KR, Fearon ER, Kern SE, Ruppert JM, Oliner JD, Kinzler KW, 
342

343

344

345

346

347

348

349

350

351

352

353

354

355

356

357

358

359

360

361

362

363

364

365

366

367

368

369

370

371

372

Vogelstein B. 1991. Scrambled exons. Cell 64(3):607-613. DOI 10.1016/00928674(91)90244-s.

Cocquerelle C, Mascrez B, Hétuin D, Bailleul B. 1993. Mis-splicing yields circular rna molecules. The FASEB Journal 7(1):155-160. DOI 10.1096/fasebj.7.1.7678559.

An Yong, Cai Huihua, Zhang Yue, Liu Shengyong, Duan Yunfei, Sun Donglin, Chen Xuemin, He Xiaozhou. 2018. circZMYM2 Competed Endogenously with miR-3355p to Regulate JMJD2C in Pancreatic Cancer. Cell Physiol Biochem 51(5): 2224-2236. DOI $10.1159 / 000495868$.

\section{Zhao J, Zou H, Han C, Ma J, Zhao J, Tang J. 2018. Circlular RNA BARD1} (Hsa_circ_0001098) overexpression in breast cancer cells with TCDD treatment could promote cell apoptosis via miR-3942/BARD1 axis. Cell Cycle 17(24):2731-2744. DOI 10.1080/15384101.2018.1556058.

Gong GH, An FM, Wang Y, Bian M, Wang D, Wei CX. 2018. Comprehensive Circular RNA Profiling Reveals the Regulatory Role of the CircRNA-0067835/miR-155 Pathway in Temporal Lobe Epilepsy. Cell Physiol Biochem 51(3):1399-1409. DOI $10.1159 / 000495589$.

Wang K, Gan TY, Li N, Liu CY, Zhou LY, Gao JN, Chen C, Yan KW, Ponnusamy M, Zhang YH, Li PF. 2017. Circular RNA mediates cardiomyocyte death via miRNAdependent upregulation of MTP18 expression. Cell Death Differ 24(6):1111-1120. DOI 10.1038/cdd.2017.61.

Fu L, Jiang Z, Li T, Hu Y, Guo J. 2018. Circular RNAs in hepatocellular carcinoma: Functions and implications. Cancer Med. [Epub ahead of print]. DOI 10.1002/cam4.1574.

Tian M, Chen R, Li T, Xiao B. 2018. Reduced expression of circRNA hsa_circ_0003159 in gastric cancer and its clinical significance. J Clin Lab Anal. 32(3). DOI 10.1002/jcla.22281.

Szabo L, Salzman J. 2016. Detecting circular RNAs: bioinformatic and experimental challenges. Nat Rev Genet. 17(11):679-692. DOI 10.1038/nrg.2016.114.

Shao Y, Li J, Lu R, Li T, Yang Y, Xiao B, Guo J. 2017. Global circular RNA expression profile of human gastric cancer and its clinical significance. Cancer Med. 6(6):11731180. DOI 10.1002/cam4.1055. 
Chen S, Li T, Zhao Q, Xiao B, Guo J. 2017. Using circular RNA hsa_circ_0000190 as a new biomarker in the diagnosis of gastric cancer. Clin Chim Acta 466:167-171. DOI 10.1016/j.cca.2017.01.025.

Zhang H, Deng T, Ge S, Liu Y, Bai M, Zhu K, Fan Q, Li J, Ning T, Tian F, Li H, Sun W, Ying G, Ba Y. 2019. Exosome circRNA secreted from adipocytes promotes the growth of hepatocellular carcinoma by targeting deubiquitination-related USP7. Oncogene 38(15):2844-2859. DOI 10.1038/s41388-018-0619-z.

Chen Z, Duan X. 2018. hsa_circ_0000177-miR-638-FZD7-Wnt Signaling Cascade Contributes to the Malignant Behaviors in Glioma. DNA Cell Biol 37(9):791-797. DOI 10.1089/dna.2018.4294.

Li P, Yang X, Yuan W, Yang C, Zhang X, Han J, Wang J, Deng X, Yang H, Li P, Tao J, Lu Q, Gu M. 2018. CircRNA-Cdr1as Exerts Anti-Oncogenic Functions in Bladder Cancer by Sponging MicroRNA-135a. Cell Physiol Biochem 46(4):1606-1616. DOI $10.1159 / 000489208$.

Bian A, Wang Y, Liu J, Wang X, Liu D, Jiang J, Ding L, Hui X. 2018. Circular RNA Complement Factor H (CFH) Promotes Glioma Progression by Sponging miR-149 and Regulating AKT1. Med Sci Monit 24:5704-5712. DOI 10.12659/MSM.910180.

Sun H, Xi P, Sun Z, Wang Q, Zhu B, Zhou J, Jin H, Zheng W, Tang W, Cao H, Cao X. 2018. Circ-SFMBT2 promotes the proliferation of gastric cancer cells through sponging miR-182-5p to enhance CREB1 expression.Cancer Manag Res 10:57255734. DOI 10.2147/CMAR.S172592.

Jin Y, Yu LL, Zhang B, Liu CF, Chen Y. 2018. Circular RNA hsa_circ_0000523 regulates the proliferation and apoptosis of colorectal cancer cells as miRNA sponge. Braz J Med Biol Res 51(12):e7811. DOI 10.1590/1414-431X20187811.

Chen F, Feng Z, Zhu J, Liu P, Yang C, Huang R, Deng Z. 2018. Emerging roles of circRNA_NEK6 targeting miR-370-3p in the proliferation and invasion of thyroid cancer via Wnt signaling pathway. Cancer Biol Ther 19(12):1139-1152. DOI 10.1080/15384047.2018.1480888.

Wang L, Wei Y, Yan Y, Wang H, Yang J, Zheng Z, Zha J, Bo P, Tang Y, Guo X, Chen W, Zhu X, Ge L. 2018. CircDOCK1 suppresses cell apoptosis via inhibition of miR-196a-5p by targeting BIRC3 in OSCC. Oncol Rep. 39(3):951-966. DOI 
AbouHaidar MG, Venkataraman S, Golshani A, Liu B, Ahmad T. 2014. Novel coding, translation, and gene expression of a replicating covalently closed circular RNA of 220 nt. Proc Natl Acad Sci U S A 111(40):14542-14547. DOI 10.1073/pnas.1402814111.

Yang Y, Fan X, Mao M, Song X, Wu P, Zhang Y, Jin Y, Yang Y, Chen LL, Wang Y, Wong CC, Xiao X, Wang Z. 2017. Extensive translation of circular RNAs driven by N6-methyladenosine. Cell Res 27(5):626-641. DOI 10.1038/cr.2017.31.

Perriman R, Ares M Jr. 1998. Circular mRNA can direct translation of extremely long repeating-sequence proteins in vivo. RNA 4(9):1047-54. DOI $10.1017 / \mathrm{s} 135583829898061 \mathrm{x}$.

Chen CY, Sarnow P. 1995. Initiation of protein synthesis by the eukaryotic translational apparatus on circular RNAs. Science 268(5209):415-7. DOI 10.1126/science.7536344.

Li Z, Huang C, Bao C, Chen L, Lin M, Wang X, Zhong G, Yu B, Hu W, Dai L, Zhu P, Chang Z, Wu Q, Zhao Y, Jia Y, Xu P, Liu H, Shan G1. 2015. Exon-intron circular RNAs regulate transcription in the nucleus.Nat Struct Mol Biol 22(3):256-64. DOI 10.1038/nsmb.2959.

Chen LL. 2016. The biogenesis and emerging roles of circular RNAs. Nat Rev Mol Cell Biol 17(4):205-11. DOI 10.1038/nrm.2015.32.

Ashwal-Fluss R, Meyer M, Pamudurti NR, Ivanov A, Bartok O, Hanan M, Evantal N, Memczak S, Rajewsky N, Kadener S. 2014. circRNA biogenesis competes with premRNA splicing. Mol Cell 56(1):55-66. DOI 10.1016/j.molcel.2014.08.019.

Du WW, Yang W, Liu E, Yang Z, Dhaliwal P, Yang BB. 2016. Foxo3 circular RNA retards cell cycle progression via forming ternary complexes with p21 and CDK2. Nucleic Acids Res 44(6):2846-58. DOI 10.1093/nar/gkw027.

Chen Y, Yang F, Fang E, Xiao W, Mei H, Li H, Li D, Song H, Wang J, Hong M, Wang X, Huang K, Zheng L, Tong Q. 2019. Circular RNA circAGO2 drives cancer progression through facilitating HuR-repressed functions of AGO2-miRNA complexes. Cell Death Differ 26(7):1346-1364. DOI 10.1038/s41418-018-0220-6.

Bray F, Ferlay J, Soerjomataram I, Siegel RL, Torre LA, Jemal A. 2018. Global cancer statistics 2018: GLOBOCAN estimates of incidence and mortality worldwide for 36 
cancers in 185 countries.CA Cancer J Clin 68(6):394-424. DOI 10.3322/caac.21492.

Zheng Q, Bao C, Guo W, Li S, Chen J, Chen B, Luo Y, Lyu D, Li Y, Shi G, Liang L, Gu J, He X, Huang S. 2016. Circular RNA profiling reveals an abundant circHIPK3 that regulates cell growth by sponging multiple miRNAs.Nat Commun 7:11215. DOI 10.1038/ncomms 11215 .

Cao Y, Yuan G, Zhang Y, Lu R. 2018. High glucose-induced circHIPK3 downregulation mediates endothelial cell injury. Biochem Biophys Res Commun 507(1-4):362-368. DOI 10.1016/j.bbrc.2018.11.041.

Yu H, Chen Y, Jiang P. 2018. Circular RNA HIPK3 exerts oncogenic properties through suppression of miR-124 in lung cancer. Biochem Biophys Res Commun 506(3):455462. DOI 10.1016/j.bbrc.2018.10.087.

\section{Li Y, Zheng F, Xiao X, Xie F, Tao D, Huang C, Liu D, Wang M, Wang L, Zeng F,} Jiang G. 2017. CircHIPK3 sponges miR-558 to suppress heparanase expression in bladder cancer cells. EMBO Rep 18(9):1646-1659. DOI 10.15252/embr.201643581.

Chi BJ, Zhao DM, Liu L, Yin XZ, Wang FF, Bi S, Gui SL, Zhou SB, Qin WB, Wu DM, Wang SQ. 2019. Downregulation of hsa_circ_0000285 serves as a prognostic biomarker for bladder cancer and is involved in cisplatin resistance. Neoplasma 66(2):197-202. DOI 10.4149/neo_2018_180318N185.

Li B, Xie F, Zheng FX, Jiang GS, Zeng FQ, Xiao XY. 2017. Overexpression of CircRNA BCRC4 regulates cell apoptosis and MicroRNA-101/EZH2 signaling in bladder cancer. J Huazhong Univ Sci Technolog Med Sci 37(6):886-890. DOI 10.1007/s11596-0171822-9.

Luo L, Gao Y, Sun X. 2018. Circ-ITCH correlates with small tumor size, decreased FIGO stage and prolonged overall survival, and it inhibits cells proliferation while promotes cells apoptosis in epithelial ovarian cancer.Cancer Biomark. 23(4):505-513. DOI 10.3233/CBM-181609.

Wang M, Chen B, Ru Z, Cong L. 2018. CircRNA circ-ITCH suppresses papillary thyroid cancer progression through miR-22-3p/CBL/ $\beta$-catenin pathway.Biochem Biophys Res Commun. 504(1):283-288. DOI 10.1016/j.bbrc.2018.08.175.

Yang C, Yuan W, Yang X, Li P, Wang J, Han J, Tao J, Li P, Yang H, Lv Q, Zhang W. 2018. Circular RNA circ-ITCH inhibits bladder cancer progression by sponging miR- 
465

466

467

468

469

470

471

472

473

474

475

476

477

478

479

480

481

482

483

484

485

486

487

488

489

490

491

492

493

494

495

17/miR-224 and regulating p21, PTEN expression.Mol Cancer. 17(1):19. DOI 10.1186/s12943-018-0771-7.

Xie F, Li Y, Wang M, Huang C, Tao D, Zheng F, Zhang H, Zeng F, Xiao X, Jiang G. 2018. Circular RNA BCRC-3 suppresses bladder cancer proliferation through miR182-5p/p27 axis. Mol Cancer. 17(1):144. DOI 10.1186/s12943-018-0892-z.

Liu H, Chen D, Bi J, Han J, Yang M, Dong W, Lin T, Huang J. 2018. Circular RNA circUBXN7 represses cell growth and invasion by sponging miR-1247-3p to enhance B4GALT3 expression in bladder cancer. Aging (Albany NY). 10(10):2606-2623. DOI 10.18632/aging.101573.

Liu H, Bi J, Dong W, Yang M, Shi J, Jiang N, Lin T, Huang J. 2018. Invasion-related circular RNA circFNDC3B inhibits bladder cancer progression through the miR-11783p/G3BP2/SRC/FAK axis. Mol Cancer. 17(1):161. DOI 10.1186/s12943-018-0908-8.

Zhong Z, Lv M, Chen J. 2016. Screening differential circular RNA expression profiles reveals the regulatory role of circTCF25-miR-103a-3p/miR-107-CDK6 pathway in bladder carcinoma. Sci Rep. 6:30919. DOI 10.1038/srep30919.

Bi J, Liu H, Cai Z, Dong W, Jiang N, Yang M, Huang J, Lin T. 2018. Circ-BPTF promotes bladder cancer progression and recurrence through the miR-31-5p/RAB27A axis.Aging (Albany NY). 10(8):1964-1976. DOI 10.18632/aging.101520.

Zeng Z, Zhou W, Duan L, Zhang J, Lu X, Jin L, Yu Y. 2019. Circular RNA circVANGL1 as a competing endogenous RNA contributes to bladder cancer progression by regulating miR-605-3p/VANGL1 pathway. J Cell Physiol. 234(4):3887-3896. DOI $10.1002 /$ jcp. 27162 .

Sun M, Zhao W, Chen Z, Li M, Li S, Wu B, Bu R. 2019. Circ_0058063 regulates CDK6 to promote bladder cancer progression by sponging miR-145-5p. J Cell Physiol. 234(4):4812-4824. DOI 10.1002/jcp.27280.

Zhong Z, Huang M, Lv M, He Y, Duan C, Zhang L, Chen J. 2017. Circular RNA MYLK as a competing endogenous RNA promotes bladder cancer progression through modulating VEGFA/VEGFR2 signaling pathway. Cancer Lett. 403:305-317. DOI 10.1016/j.canlet.2017.06.027.

Huang W, Lu Y, Wang F, Huang X, Yu Z. 2018. Downregulation of circular RNA hsa_circ_0000144 inhibits bladder cancer progression via stimulating miR-217 and 
suppressing RUNX2 expression.Gene. 678:337-342. DOI 10.1016/j.gene.2018.08.036.

497

498

499

500

501

502

503

504

505

506

507

508

509

510

511

512

513

514

515

516

517

518

519

520

521

522

523

524

525

526
Yang C, Wu S, Wu X, Zhou X, Jin S, Jiang H. 2019. Silencing circular RNA UVRAG inhibits bladder cancer growth and metastasis by targeting the microRNA223/fibroblast growth factor receptor 2 axis. Cancer Sci. 110(1):99-106. DOI 10.1111/cas.13857.

Xiong Y, Zhang J, Song C. 2019. CircRNA ZNF609 functions as a competitive endogenous RNA to regulate FOXP4 expression by sponging miR-138-5p in renal carcinoma. J Cell Physiol 234(7):10646-10654. DOI 10.1002/jcp.27744.

Wang G, Xue W, Jian W, Liu P, Wang Z, Wang C, Li H, Yu Y, Zhang D, Zhang C. 2018. The effect of Hsa_circ_0001451 in clear cell renal cell carcinoma cells and its relationship with clinicopathological features. J Cancer. 9:3269-3277. DOI 10.7150/jca.25902.

Han Z, Zhang Y, Sun Y, Chen J, Chang C, Wang X, Yeh S. 2018. ER $\beta$-Mediated Alteration of circATP2B1 and miR-204-3p Signaling Promotes Invasion of Clear Cell Renal Cell Carcinoma.Cancer Res. 78(10):2550-2563. DOI 10.1158/0008-5472.CAN$17-1575$.

Wang K, Sun Y, Tao W, Fei X, Chang C. 2017. Androgen receptor (AR) promotes clear cell renal cell carcinoma (ccRCC) migration and invasion via altering the circHIAT1/miR-195-5p/29a-3p/29c-3p/CDC42 signals.Cancer Lett. 394:1-12. DOI 10.1016/j.canlet.2016.12.036.

He JH, Han ZP, Zhou JB, Chen WM, Lv YB, He ML, Li YG. 2018. MiR-145 affected the circular RNA expression in prostate cancer LNCaP cells. J Cell Biochem. 119(11):9168-9177. DOI 10.1002/jcb.27181.

Dai Y, Li D, Chen X, Tan X, Gu J, Chen M, Zhang X. 2018. Circular RNA Myosin Light Chain Kinase (MYLK) Promotes Prostate Cancer Progression through Modulating Mir-29a Expression.Med Sci Monit. 24:3462-3471. DOI 10.12659/MSM.908009.

Kong Z, Wan X, Zhang Y, Zhang P, Zhang Y, Zhang X, Qi X, Wu H, Huang J, Li Y. 2017. Androgen-responsive circular RNA circSMARCA5 is up-regulated and promotes cell proliferation in prostate cancer. Biochem Biophys Res Commun. 493(3):1217-1223. DOI 10.1016/j.bbrc.2017.07.162. 
527

528

529

530

531

532

533

534

535

536

537

538

539

Peer] reviewing PDF | (2019:10:42273:1:0:NEW 3 Jan 2020) 


\section{Figure 1}

Locations and functions of circRNAs.

(A) Primarily, circRNAs are located in the cytoplasm, but some reside in subcellular compartments such as exosomes and mitochondria. (B) Function of circRNAs. circRNAs serve as sponges that directly bind miRNA or protein to regulate gene expression and activate signaling pathways, thus participating in the development of bladder, renal, and prostate cancers.

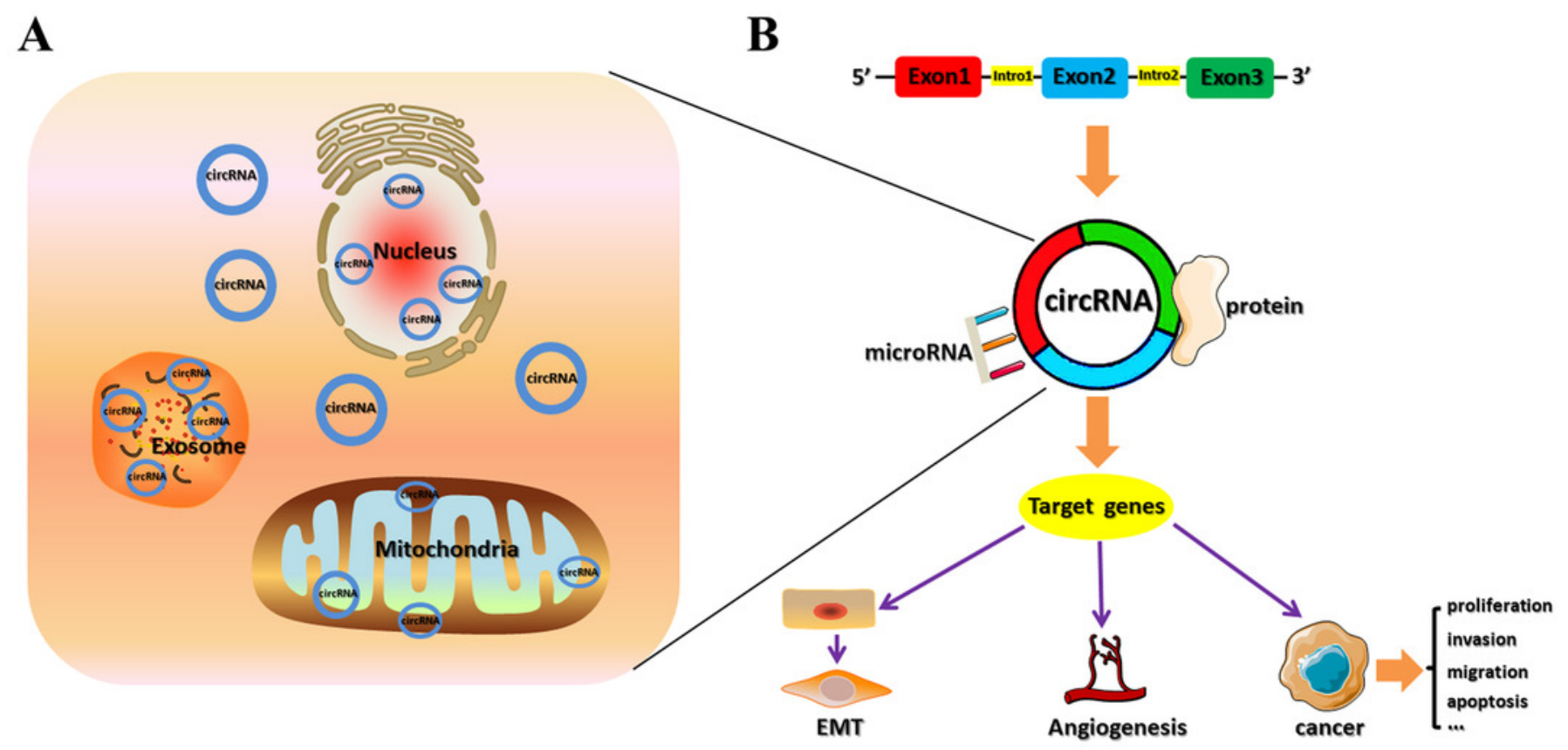




\section{Table $\mathbf{1}$ (on next page)}

Summary of circRNAs in bladder, renal, and prostate cancers. 


\begin{tabular}{|c|c|c|c|c|c|}
\hline & circRNA & miRNA & Downstream & Function & Reference \\
\hline \multirow[t]{5}{*}{$\begin{array}{l}\text { Bladder } \\
\text { cancer }\end{array}$} & circ-HIPK3 & $\operatorname{miR}-558$ & $\begin{array}{l}\text { HPSE, VEGF, } \\
\text { MMP9 }\end{array}$ & $\begin{array}{l}\text { Inhibits migration, } \\
\text { invasion, and } \\
\text { angiogenesis of } \\
\text { cancer cells in vitro, } \\
\text { as well as tumor } \\
\text { growth and } \\
\text { metastasis in vivo. }\end{array}$ & $\underset{2017}{\text { Li et al., }}$ \\
\hline & circ-BCRC4 & miR-101 & $\mathrm{EZH} 2$ & $\begin{array}{l}\text { Attenuates } \\
\text { proliferation of } \\
\text { cancer cells and } \\
\text { induces apoptosis. }\end{array}$ & $\begin{array}{l}\text { Li et } \\
2017\end{array}$ \\
\hline & circ-ITCH & $\begin{array}{l}\text { miR-17 miR- } \\
224\end{array}$ & P21, PTEN & $\begin{array}{l}\text { Induces G1 cell } \\
\text { cycle arrest and } \\
\text { apoptosis, inhibiting } \\
\text { BC } \\
\text { proliferation, } \\
\text { invasion, } \\
\text { migration in vitro, } \\
\text { as well as tumor } \\
\text { growth in vivo. }\end{array}$ & $\begin{array}{l}\text { Yang et al., } \\
2018\end{array}$ \\
\hline & circ-BCRC-3 & $\operatorname{miR}-182-5 p$ & P27 & $\begin{array}{l}\text { Attenuates } \\
\text { proliferation, cell } \\
\text { cycle progression of } \\
\mathrm{BC} \text { cell in vitro; } \\
\text { inhibits tumor } \\
\text { growth in vivo. }\end{array}$ & $\begin{array}{l}\text { Xie et al., } \\
2018\end{array}$ \\
\hline & circ-UBXN7 & miR-1247-3p & B4GALT3 & $\begin{array}{l}\text { Inhibits cancer cell } \\
\text { proliferation, } \\
\text { invasion, } \\
\text { migration in vitro; }\end{array}$ & $\underset{2018}{\text { Liu et al., }}$ \\
\hline
\end{tabular}




\begin{tabular}{|c|c|c|c|c|}
\hline \multirow[b]{3}{*}{ circ-FNDC3B } & \multirow[b]{3}{*}{$\operatorname{miR}-1178-3 p$} & \multirow[b]{3}{*}{ G3BP2 } & \multirow{2}{*}{\multicolumn{2}{|c|}{$\begin{array}{l}\text { represses tumor } \\
\text { growth in vivo. }\end{array}$}} \\
\hline & & & & \\
\hline & & & $\begin{array}{l}\text { Inhibits cancer cell } \\
\text { proliferation, } \\
\text { migration, invasion, } \\
\text { tumor growth, and } \\
\text { lymphatic metastasis } \\
\text { in vitro and in vivo. }\end{array}$ & $\underset{2018}{\text { Liu }}$ et al., \\
\hline circ-TCF25 & $\begin{array}{l}\operatorname{miR}-103 a-3 p \\
\text { and miR-107 }\end{array}$ & CDK6 & $\begin{array}{l}\text { Promotes cancer cell } \\
\text { proliferation, } \\
\text { migration in vitro, } \\
\text { tumor growth, and } \\
\text { metastasis in vivo. }\end{array}$ & $\begin{array}{l}\text { Zhong et al., } \\
2016\end{array}$ \\
\hline circ-BPTF & $\operatorname{miR}-31-5 p$ & RAB27A & $\begin{array}{l}\text { Promotes cancer cell } \\
\text { invasion, migration } \\
\text { in vitro, and tumor } \\
\text { growth in vivo. }\end{array}$ & $\begin{array}{l}\text { Bi et al., } \\
2018\end{array}$ \\
\hline circ-VANGL1 & $\operatorname{miR}-605-3 p$ & VANGL1 & $\begin{array}{l}\text { Promotes cancer cell } \\
\text { proliferation, } \\
\text { migration, } \\
\text { metastasis, cell } \\
\text { cycle progression in } \\
\text { vitro, and tumor } \\
\text { growth in vivo. }\end{array}$ & $\begin{array}{l}\text { Zeng et al., } \\
2019\end{array}$ \\
\hline circ-0058063 & $\operatorname{miR}-145-5 p$ & CDK6 & $\begin{array}{l}\text { Promotes cancer cell } \\
\text { proliferation, } \\
\text { migration, } \\
\text { metastasis, and cell } \\
\text { cycle progression; } \\
\text { inhibits apoptosis in } \\
\text { vitro. }\end{array}$ & $\begin{array}{l}\text { Sun et al., } \\
2019\end{array}$ \\
\hline circ-MYLK & miR-29a & CD31, S100A4, & Promotes cancer cell & Zhong et al., \\
\hline
\end{tabular}




\begin{tabular}{|c|c|c|c|c|c|}
\hline & & & $\begin{array}{l}\text { ZO-1, VEGFA, } \\
\text { Ras, p-Raf-1, p- } \\
\text { MEK1/2, p- } \\
\text { ERK1/2 }\end{array}$ & $\begin{array}{l}\text { proliferation, } \\
\text { invasion, and } \\
\text { migration, inhibiting } \\
\text { apoptosis in vitro; } \\
\text { facilitates tumor } \\
\text { growth, EMT, and } \\
\text { metastasis in vivo. }\end{array}$ & 2017 \\
\hline \multirow{5}{*}{$\begin{array}{l}\text { Renal } \\
\text { cell } \\
\text { cancer }\end{array}$} & circ-0000144 & miR-217 & RUNX2 & $\begin{array}{l}\text { Promotes cancer cell } \\
\text { proliferation, } \\
\text { invasion, and } \\
\text { migration in vitro, } \\
\text { as well as tumor } \\
\text { growth in vivo. }\end{array}$ & $\begin{array}{l}\text { Huang et al., } \\
2018\end{array}$ \\
\hline & circ-UVRAG & miR-223 & FGFR2 & $\begin{array}{l}\text { Promotes cancer cell } \\
\text { proliferation, } \\
\text { migration, and } \\
\text { metastasis in vitro, } \\
\text { as well as tumor } \\
\text { growth in vivo. }\end{array}$ & $\begin{array}{l}\text { Yang et al., } \\
2019\end{array}$ \\
\hline & circ- 0001451 & - & - & $\begin{array}{l}\text { Suppresses cancer } \\
\text { cell proliferation; } \\
\text { promotes apoptosis } \\
\text { in vitro. }\end{array}$ & $\begin{array}{l}\text { Wang et al., } \\
2018\end{array}$ \\
\hline & circ-ATP2B1 & $\operatorname{miR}-204-3 p$ & FN1 & $\begin{array}{l}\text { Suppresses cancer } \\
\text { cell invasion in vitro } \\
\text { and tumor growth in } \\
\text { vivo. }\end{array}$ & $\begin{array}{l}\text { Han et al., } \\
2018\end{array}$ \\
\hline & circ-HIAT1 & $\begin{array}{l}\operatorname{miR}-195-5 p \\
\operatorname{miR}-29 a-3 p \\
\operatorname{miR}-29 c-3 p\end{array}$ & CDC42 & $\begin{array}{l}\text { Suppresses cancer } \\
\text { cell invasion and } \\
\text { migration in vitro, } \\
\text { as well as tumor } \\
\text { growth in vivo. }\end{array}$ & $\begin{array}{l}\text { Wang et al., } \\
2017\end{array}$ \\
\hline
\end{tabular}




\begin{tabular}{|c|c|c|c|c|c|}
\hline \multirow[t]{2}{*}{$\begin{array}{l}\text { Prostate } \\
\text { cancer }\end{array}$} & circ-MYLK & $\operatorname{miR}-29 a$ & - & $\begin{array}{l}\text { Promotes cancer cell } \\
\text { proliferation, } \\
\text { invasion, and } \\
\text { migration; decreases } \\
\text { apoptosis in vitro. }\end{array}$ & $\begin{array}{l}\text { Dai et al., } \\
2018\end{array}$ \\
\hline & circ-SMARCA5 & - & - & $\begin{array}{l}\text { Promotes cancer cell } \\
\text { proliferation and } \\
\text { cell cycle } \\
\text { progression in vitro. }\end{array}$ & $\begin{array}{l}\text { Kong et al., } \\
2017\end{array}$ \\
\hline
\end{tabular}

